J. Clin. Chem. Clin. Biochem.

Vol. 16, 1978, pp. 235-238

\title{
Die Bestimmung von Glucose im Kapillarblut mit dem autơmatic clinical analyzer (aca) Dupont
}

\author{
Von G. Heinemann
}

Institut für Klinische Chemie, (Vorstand: Prof. Dr. med. H. Schievelbein), Deutsches Herzzentrum München

(Eingegangen am 21. September/7. November 1977)

Zusammenfassung: Es wird die Bestimmung von Glucose im Kapillarblut nach Enteiweißung auf dem aca beschrieben. Das Verfahren, dem die Hexokinase/Glucose-6-phosphatdehydrogenase-Reaktion zugrundeliegt, wird mit der Glucosedehydrogenase-Methode verglichen. Die Untersuchung zeigt, daß die mit beiden Methoden ermittelten Glucosewerte im Kapillarblut sehr gut übereinstimmen. Die Kriterien der Zuverlässigkeit werden von diesem Verfahren erfültt, so daß seine Anwendung für Einzelanalysen empfohlen werden kann. Die Bestimmung wird durch Hyperhämolyse, Hypc i vilirubinämie und Hypertriglyceridämie nicht beeinträchtigt.

\section{The determination of capillary blood glucose using the automatic clinical analyzer (aca) Dupont}

Summary: The determination of capillary blood glucose after deproteinization using the aca is described. The method, which uses the hexokinase/glucose-6-phosphate dehydrogenase reaction, is compared with the glucose dehydrogenase method. The comparison shows that glucose values measured in capillary blood are essentially the same in both methods. The requirements for quality control are fulfilled. The method is not influenced by hemolysis, bilirubinemia, and hypertriglyceridemia.

\section{Einleitung}

Die Bestimmung der Glucose mit dem aca ist bislang lediglich für Serum, Harn und Liquor beschrieben (1). Bei diesen Bestimmungen können jedoch Hyperhämolyse und/oder -bilirubinämie zu falsch negativen Ergebnissen führen $(2,3)$.

Wir stellten uns die Aufgabe, die Bestimmung der Glucose im Kapillarblut nach Enteiweißung auch auf dem aca zu ermöglichen. Folgende Gründe veranlaßten uns zu diesem Vorgehen:

1. Für Blut-Glucosebestimmungen ist die KapillarblutEntnahme die Methode der Wahl,

2. Glucosebestimmungen im Liquor sind in unserer Klinik sehr selten und für Harnżucker-Bestimmungen besteht keine Vordringlichkeit einer schnellen Befundermittlung,

3. die in unserer Klinik häufigen Glucosebestimmungen àls Notfall- und damit Einzelanalysen.

Nach Möglichkeit sollten bei der von uns angestrebten Modifikation die oben erwähnten Nachteile behoben werden.

Die Zuverlässigkeit des neuen Verfahrens und seine Korrelation zu einer anderen spezifischen Methode sollten geprüft werden.
Material und Methoden

Reagenzien

1. Triethanolamin-Hydrochlorid (Boehringer Mannheim), Nr. 127426.

2. Perchlorsäure/Perchloratlösung (Merck), Enteiweißungsmittel für die Glucosebestimmung mit Glucosedehydrogenase, Nr. 9431.

3. Testpackung „Merckotest Glucose“ (GlucosedehydrogenaseMethode) UV-Test.

4. Glucose-Standardlösungen (Merck), Nr. 9423.

5. Kontrollseren

Bilirubin Control, lyophilisiertes Bilirubin-Kontrollserum (Merz- u. Dade); Elevated Chemistry Control, lyophilisiertes Kontrollserum (du Pont de Nemours and Co); Precinorm S, flüssiges Kontrollserum (Boehringer Mannheim)

\section{Geräte}

1. aca (automatic clinical analyzer), Fa. du Pont de Nemours and Co., Wilmington, Del 19898

2. Eppendorf Substratmeßplatz

3. Eppendorf Mikrolitersystem

4. 2. Eppendorf Probe-Reagenz-Dosierer Nr. 5231

5. EDTA-diKalium + Natriumjodacetat-Blutzuckerrührchen 113 ENJ, Fa. Greiner

6. Accupetten (Einmalkapillarpipetten) $100 \mu \mathrm{l}, \mathrm{Fa}$. Merz u. Dade

7. pH-Meter 801, Fa. Orion Research

\section{Löșungen}

1. Triethanolamin-Hydrochlorid-Puffer $0,1 \mathrm{~mol} / 1, \mathrm{pH} 7,8$. Es wird eine wäßrige Puffer-Stammlösung, $1 \mathrm{~mol} / 1$ hergestellt, die je nach Bedarf $1: 10$ mit bidest. Wasser weiterverdünnt und mit $10 \mathrm{~mol} / 1 \mathrm{NaOH}$ auf $\mathrm{pH} \mathrm{7,8} \mathrm{eingestellt} \mathrm{wird.}$ 
2. Herstellung der Lösungen für „Merckotest Glucose“ laut Testvorschrift.

\section{Bestimmungsmaterial und Untersuchungsgang}

Für die Glucosebestimmung wird Kapillarblut verwendet. Dazu wird die Accupette über die $100 \mu \mathrm{l}$-Ringmarkierung hinaus bis zum blauen Farbring mit Blut gefüllt und anschließend sofort in ein Blutzuckerröhrchen entleert, so daß sich etwa $140 \mu$ l Blut auf dem Boden des Röhrchens befinden. Das Röhrchen wird leicht schräg gehalten und mehrfach gedreht, um das Blut mit dem Gerinnungs- und Glykolysehemmer zu vermischen. Danạch werden mit einem Probe-Reagenz-Dosierer 0,05 ml Blut entnommen und mit $0,5 \mathrm{ml}$ Perchlorsäure/Perchloratlösung zur Enteiweißung versetzt, kurz geschüttelt, $2 \mathrm{~min}$ bei etwa $10.000 \mathrm{~g}$ zentrifugiert und der eiweißfreie Überstand zur Bestimmung eingesetzt. Um den Einfluß von Hämolyse und Hyperbilirubinämie auf die modifizierte Methode zu prüfen, wurden Hämolysat und Bilirubin in steigenden Konzentrationen verschiedenen Seren zugesetzt. Für den Bilirubinzusatz benutzten wir Bilirubin-Control (Merz u. Dade).

\section{Bestimmung auf dem Substratmeßplatz}

$0,05 \mathrm{ml}$ Überstand werden mit Hilfe eines Probe-ReagenzDosierers mit 0,5 ml Reagenzlösung des Merckotests Glucose versetzt und $15 \mathrm{~min}$ bei $25^{\circ} \mathrm{C}$ inkubiert. Dabei wird $\beta$ - $D$-Glucose durch Glucosedehydrogenase zu Gluconsäurelacton dehydriert und NAD zu NADH reduziert (4). Anschließend wird die $\mathrm{Ab}$ sorption der Probe gegen einen Reagenzienleerwert bei $334 \mathrm{~nm}$ gemessen.

\section{Bestimmung auf dem aca}

\section{aca-Originalmethode}

Die aca-Glucosemethode ist eine Adaptation der HexokinaseGlucose-6-phosphatdehydrogenase-Methode, die zuerst von Schmidt (5) publiziert wurde, mit NADP anstelle von NAD als Coenzym. Die Reagenzien befinden sich in Einmal-Testpackungen in trockener Form und in verschiedenen Kompartimenten. Im Gerät werden dann 0,040 ml Probe (Serum, Harn, Liquor) mit 4,96 ml bidest. Wasser verdünnt und in eine Reagenz-Testpackung injiziert. Nach einer Inkubation der Testpackung von $6 \mathrm{~min}$ bei $37^{\circ} \mathrm{C}$ wird die Absorption bei zwei Wellenlängen ( 340 und $385 \mathrm{~nm}$ ) gemessen und die Differenz zur Berechnung der Glucosekonzentration benutzt.

\section{Modifizierte aca-Methode}

Der stark saure Enteiweißungs-Überstand wurde durch Zusatz eines geeigneten Puffers in einen schwach alkalischen Bereich übergeführt und die bei der Enteiweißung entstehende Probenverdünnung ausgeglichen.

1. Auf dem freien Pufferplatz Nr. 6 wurde ein Kanister mit Triethanolamin-Hydrochlorid $0,1 \mathrm{~mol} / \mathrm{l}, \mathrm{pH} \mathrm{7,8}$ angeschlossen,

2. die Zufuhr des Puffers zur Probenverdünnung wurde von Pufferplatz Nr. 1 auf Platz Nr. 6 umprogrammiert,

3. das Probe-Ansaugvolumen auf Kanal 1 wurde von $0,040 \mathrm{ml}$ auf $0,240 \mathrm{ml}$ erhöht und damit die Puffermenge auf $4,76 \mathrm{ml}$ vermindert,

4. der vorläufige Skalenfaktor wurde mit 2 multipliziert und eingegeben,

5. der vorläufige Startpunkt wurde zunächst beibehalten.

Die unter Punkt 3 und 4 angeführten Maßnahmen gleichen die Verdünnung durch die Enteiweißung aus.

Für die Kalibrierung des aca wurde Vollblut mit niedriger (etwa 2,5 mmol/l), mittlerer (etwa 13-17 mmol/l) und hoher (24-27 $\mathrm{mmol} / \mathrm{l}$ ) Glucosekonzentration in genügender Menge enteiweißt, so daß aus den drei enteiweißten Ửberständen Dreifach-Bestimmungen sowohl auf dem aca wie auch auf dem Substratmeßplatz möglich waren. Die Mittelwerte wurden zur Berechnung des endgültigen Startpunktes und Skalenfaktors benutzt. Nach dieser Kalibrierung haben wir im unverdünnten Kontrollserum Elevated Chemical Control und in einer $1: 2$ und $1: 10$ Verdünnung die Glucosekonzentrationen mehrfach bestimmt. Die Meßwerte der Kontrollseren gleicher Charge können dann in $\mathrm{Zu}$ kunft anstelle von Blut als Sollwerte zur Kalibrierung verwendet werden.

\section{Ergebnisse}

\section{Konzentration und $\mathrm{pH}-\mathrm{Wert}$ des Puffers}

In Vorversuchen konnte für eine ausreichende Pufferung des enteiweißten Überstandes $0,1 \mathrm{~mol} / 1$ TriethanolaminHydrochlorid ermittelt werden. Der pH-Wert des Puffers von 7,8 wurde gewählt , da er dem pH des Probe-Reagenzgemisches in der aca-Testpackung bei Verwendung von Serum entsprach; denn auch bei $\mathrm{pH}$-Werten des Puffers zwischen 7,6 und 8,1 war eine Beeinträchtigung der Reaktion nicht festzustellen.

\section{Linearität}

Die Linearität wurde mit wäßrigen Glucose-Standardlösungen (Glucose-Konzentration 1,$38 ; 2,77 ; 5,55$; $11,10 ; 16,65 ; 27,75 \mathrm{mmol} / 1)$ überprüft. Die wäßrigen Standards wurden gleichfalls mit Perchlorsäure/Perchlorat im Verhältnis $1: 11$ verdünnt. Linearität ergab sich über den gesamten Bereich von 0-27,75 mmol/1 Glucose (Abb. 1). Die Punkte der Geraden entsprechen Mittelwerten aus Doppelbestimmungen.

\section{Präzision in der Serie}

Es wird die Repetierbarkeit der Methode durch Glucosebestimmungen in je 20 enteiweißten Überständen mit niedriger und erhöhter Glucose-Konzentration überprüft. Die Ergebnisse sind in Tabelle 1 dargestellt.

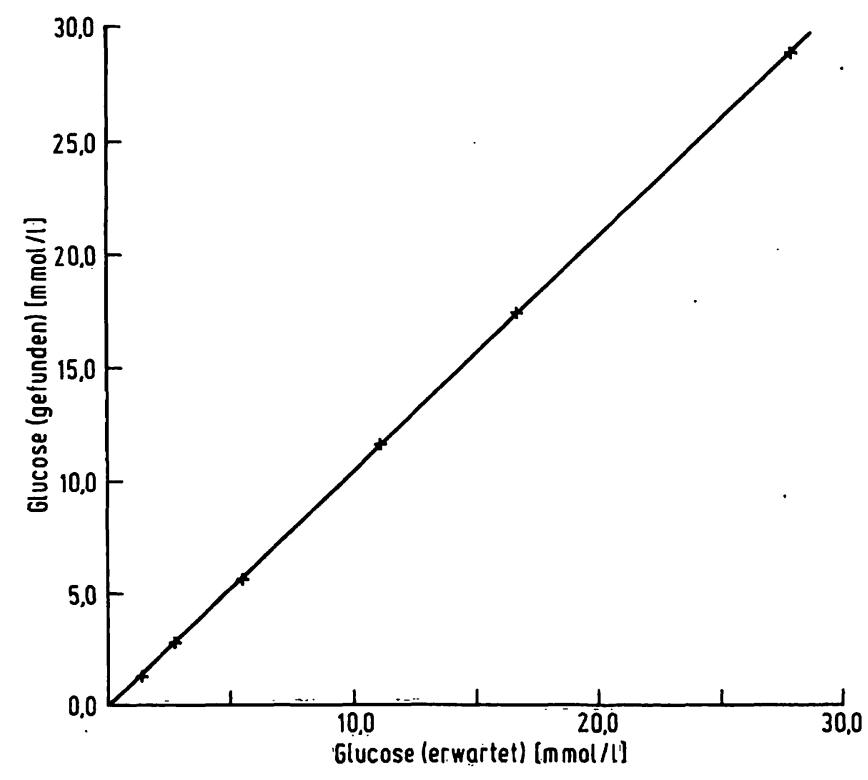

Abb. 1. Darstellung des Linearitätsbereichs bis $27,75 \mathrm{mmol} / 1$ Glucose. Sollwerte der Standardlösungen $(x)$, aca-Meßwerte $(y)$. Gerade: $y=1,04 x-0,06, x=0,99$.

Tab. 1. Repetierbarkeit der Glucosebestimmung in Kapillarblut nach Enteiweißung mit niedriger und erhöhter GlucoseKonzentration

\begin{tabular}{lllll}
\hline $\begin{array}{l}\text { n Mittelwert } \bar{x} \\
\text { mmol/1 } \\
0\end{array}$ & $\begin{array}{l} \pm s \\
\text { mmol/1 }\end{array}$ & $\begin{array}{l}\text { Konzentration } \\
\text { mmol/1 } \\
\text { höchste }\end{array}$ & niedrigste & $\begin{array}{l}\text { VK } \\
\%\end{array}$ \\
\hline $20 \quad 2,15$ & 0,07 & 2,30 & 2.05 & 3,41 \\
2016,34 & 0,28 & 17,00 & 15,81 & 1,73 \\
\hline
\end{tabular}




\section{Präzision von Tag zu Tag}

Die Präzision von Tag zu Tag wurde an 23 Tagen mit Precinorm $S$ geprüft und erbrachte bei einer GlucoseKonzentration von $\bar{x}=5,58 \mathrm{mmol} / 1$ einen Variationskoeffizienten (VK\%) von 2,93 .

\section{Richtigkeit}

Zur Prüfung der Richtigkeit führten wir Vergleichsversuche durch. Die Glucose-Konzentrationen der enteiweißten Überstände wurden sowohl auf dem aca wie auch mit der Glucosedehydrogenase-Methode auf einem Eppendorf Substratmeßplatz als Doppelbestimmung ermittelt. Wie Abbildung 2 zeigt, fanden sich mit beiden Methoden sehr gut übereinstimmende Werte. Der Korrelationskoeffizient für $\mathrm{N}=52$ betrug $\mathrm{r}=0,9997$, die Regressionsgerade $y=0,9953 x+0,0062$ und die Streuung $s_{y . x}= \pm 0,636 \mathrm{mmol} / 1$.

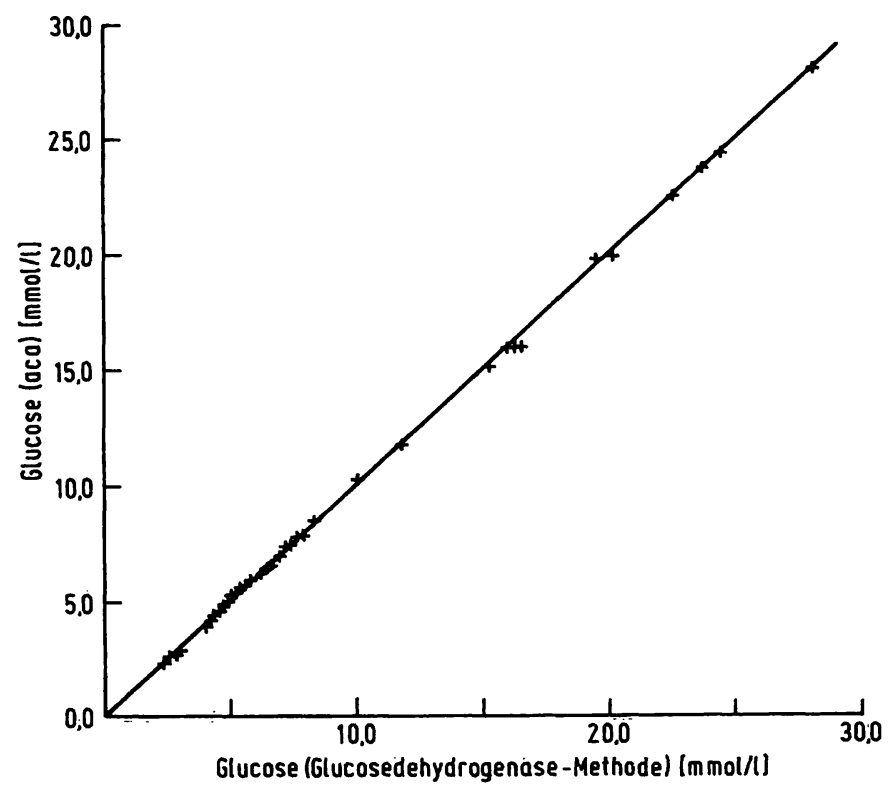

Abb. 2. Vergleich zwischen der für den aca entwickelten Methode und der Glucose-Dehydrogenase-UV-Methode.

\section{Interferenzen}

Beim aca-Originalverfahren zur Bestimmung der Glucose in Serum interferieren Hyperhämolyse und/oder -bilinubinämie je nach Schweregrad.

İn den Kliniken des Deutschen Herzzentrums München fallen gehäuft hämolytische und ikterische Blutproben an (Einsatz von Herz-Lungenmaschine, Herzklappenambulanz). Wir haben daher die beschriebene Bestimmung anhand von hämolytischen und ikterischen Blutproben überprüft. Wir fanden keinerlei Störung, auch nicht bei sehr hohen Bilirubin- und Hämoglobinkonzentrationen im Plasma (Tab. 2). Eine Vermehrung von prä- $\beta$-Lipoproteinen stört die Bestimmungen ebenfalls nicht, da sie bẹi der Enteiweißung präzipitieren.
Tab. 2. Vergleich von Glucose-Konzentrationen im Serum vor (a) und nach (b) Zusatz von Hämolysat bzw. Bilirubin

\begin{tabular}{|c|c|c|c|c|c|}
\hline \multirow[t]{2}{*}{$\begin{array}{l}\text { Hämoglobin } \\
\text { im Serum } \\
(\mathrm{g} / 1)\end{array}$} & $\begin{array}{l}\text { Glucos } \\
\text { (mmol }\end{array}$ & m Serum & \multirow[t]{2}{*}{$\begin{array}{l}\text { Bilirubin } \\
\text { im Serum } \\
(\mu \mathrm{mol} / 1)\end{array}$} & $\begin{array}{l}\text { Gluce } \\
\text { (mmc }\end{array}$ & m Serum \\
\hline & $\mathbf{a}$ & b & & a & b \\
\hline $\begin{array}{l}0.7 \\
1,4 \\
2,8 \\
4,2\end{array}$ & $\begin{array}{l}7,19 \\
7,17 \\
7,02 \\
7,06\end{array}$ & $\begin{array}{l}7,14 \\
7,23 \\
7,05 \\
7,00\end{array}$ & $\begin{array}{r}63 \\
91 \\
176 \\
206 \\
259\end{array}$ & $\begin{array}{l}4,83 \\
4,06 \\
2,60 \\
4,98 \\
5,11\end{array}$ & $\begin{array}{l}4,86 \\
4,03 \\
2,61 \\
4,99 \\
5,15\end{array}$ \\
\hline $\begin{array}{r}14,0 \\
18,0 \\
140,0\end{array}$ & $\begin{array}{r}6,59 \\
6,06 \\
28,06\end{array}$ & $\begin{array}{r}6,64 \\
5,99 \\
27,88\end{array}$ & 307 & 5,21 & 5,22 \\
\hline
\end{tabular}

Der in der Kardiologie zur Messung der Herz-Kreislaufzeit verwendete Farbstoff Indozyanin-Grün. stört wegen seiner geringen Konzentration im Blut die Bestimmung ebenfalls nicht.

\section{Normbereich}

Die durch die Kalibrierung des aca erzielte Úbereinstimmung der Meßwerte beider Methoden gestattet es, den für die Glucosedehydrogenase-Methode ermittelten Bereich von 2,77-5,55 mmol/1 (4) Glucose für Kapillarblut zu verwenden.

\section{Diskussion}

Beim Vergleich der Zuverlässigkeitskriterien zeigen die Ergebnisse der Modifikation und diejenigen der acaOriginalmethode (6) wie auch die der Glucosedehydrogenase-Methode (4) eine gute Úbereinstimmung. Das Ergebnis des Methodenvergleichs in Abbildung 2 verdeutlicht die Richtigkeit im Bereich bis 27,75 mmol/1. Die nun ermöglichte Glucosebestimmung im Kapillarblut auf dem aca kommt damit auch den erneuten Empfehlungen der Deutschen Diabetesgesellschaft entgegen, Kapillarblut zur Diabetesdiagnostik zu verwenden (7).

Als wesentliche Bereicherung der Modifikation gegenüber der aca-Originalmethode betrachten wir, zumindest für unseren Bereich, die durch die Enteiweißung wiedergewonnene Unempfindlichkeit der Methode gegenüber Hyperhämolysen und Hyperbilirubinämien. Weiterhin bleibt auch der dem Pflegepersonal vertraute Vorgang der Kapillarblutentnahme und die Handhabung des Zuckerröhrchens gleich. Die Frage des Normbereichs wird nicht berüht, da keine Änderung eintritt.

Die beschriebene Modifikation wird seit einigen Monaten in unserem Zentrallabor verwendet. Sie hat sich bewährt und wird bei Notfall-Untersuchungen durchgeführt; bei Serienmessungen bevorzugen wir aus ökonomischen Gründen den Substratmeßplatz. 


\section{Literatur}

1. Test Methodology Glucose in Chemistry Instruction Manual, du Pont Corp., Wilmington, Del. 19898, (1975),

2. Gochman, N., Ryan, W. T., Sterling, R. E. \& Widdowson, G. M. (1975), Clin. Chem. 21, 356-361.

3. Carey, R. N., Feldbruegge, D. \& Westgard, O. (1974), Clin. Chem. 20, 595-602.

4. Banauch, D., Brümmer, W., Ebeling, W., Metz, H., Rindfrey, H., Lang, H., Leybold, K. \& Rick, W. (1975), diese Z. 13, 101-107.

5. Schmidt, F. H. (1961), Klin. Wochenschr. 39, 1244-1247.

6. Westgard, J. O. \& Lahmeyer, B. L. (1972), Clin. Chem. 18, $340-348$.

7. Jahrestagung der Deutschen Diabetesgesellschaft, Bad Homburg (1977).
Dr. G. Heinemann

Institut fur Klinische Chemie Deutsches Herzzentrum München Lothstr. 11

8000 München 2 